Voix et Images

volxetimages

\title{
Kamalmouk ou le rêve de Marius Barbeau
}

\section{Jean Morisset}

Volume 21, numéro 2 (62), hiver 1996

Suzanne Jacob

URI : https://id.erudit.org/iderudit/201245ar

DOI : https://doi.org/10.7202/201245ar

Aller au sommaire du numéro

Éditeur(s)

Université du Québec à Montréal

ISSN

0318-9201 (imprimé)

1705-933X (numérique)

Découvrir la revue

Citer cet article

Morisset, J. (1996). Kamalmouk ou le rêve de Marius Barbeau. Voix et Images, 21(2), 352-364. https://doi.org/10.7202/201245ar d'utilisation que vous pouvez consulter en ligne.

https://apropos.erudit.org/fr/usagers/politique-dutilisation/ 


\title{
Kamalmouk ou le rêve de Marius Barbeau
}

\author{
Jean Morisset, Université du Québec à Montréal
}

à ma collègue Sylvie Vincent qui tente de parcourir avec une telle ardeur les romans non paginés de la littérature orale.

Après mûre réflexion, Beach tira un carnet de son paqueton, écrivit quelques lignes sur une feuille blanche [...], la remit à Rayon-de-Soleil, avec le soin qu'on donne à une chose précieuse. Les sauvages ont un respect étrange pour toute écriture...

Le Rêve de Kamalmouk, p. 166

Les sauvages ont un respect étrange pour toute écriture!... Celui qu'éprouve, bien sûr, tout être analphabète vis-à-vis de ces signes cabalistiques déposés sur un étrange morceau d'écorce préfabriqué. Morceau si mince et si blanc qu'on n'arrive plus à savoir de quel arbre il provient, de quelle peau tannée il dérive ou de quelle tribu il est issu. Et tous ces troncs-pelés venus y déposer de curieuses graphies à l'aide de ce minuscule instrument qui verse du sang noir ou bleu..., tous ces dos-blancs possèdent un art magique, plus magique encore, semble-t-il, que celui des ceintures de wampum.

Avec les quelque six ou sept cents écrits qu'il a publiés et les dizaines de milliers de pages qu'il a colligées, Marius Barbeau n'était certes ni sauvage ni analphabète, mais il savait! Ayant, en effet, consacré toute son œuvre à réfléchir à la sensibilité analphabète et à vouloir transcrire la pensée orale, il savait donc d'instinct quelque chose qu'il n'avait ni à préciser ni à légitimer, quelque chose s'inscrivant au-delà de toute écriture et sur lequel j'aimerais m'interroger.

En parcourant rapidement cette forêt de textes et les multiples sentiers qui la sillonnent, Barbeau apparaît d'emblée comme un géant. Une espèce de Riopelle de la pensée diffuse de ce Canada pré-Québec dont on a à peine commencé à questionner et la trajectoire et le sens. Un 
géant, ai-je dit! Certes, mais tout comme les Ouinabojos et les Oiseauxde-Feu hantant le vieux socle précambrien, depuis le lac Supérieur jusqu'au lac Contwoyto, et bien au-delà, il s'agit d'un géant endormi, dont on arrive mal à départager l'œuvre et la vie, la pensée et le désir.

Peut-être l'auteur d'ouvrages apparemment aussi divers et disparates que Pathfinders in the North Pacific et les Contes du Grand-Père SeptHeures, les Tsimsyan Myths et Les Saintes Artisanes, les Totem Poles et le Kingdom of the Saguenay, L'Homme aux trois femmes et les Indian Days in the Canadian Rockies ou... les Medecine-Men et L'île d'Orléans était-il aussi un peu magicien, c'est le moins qu'on puisse dire. Un magicien qui, cependant, se sera sans cesse retenu d'exprimer de façon trop directe son univers intérieur.

On considère que l'œuvre de Marius Barbeau se divise en deux "systèmes claniques séparés": un clan autochtone, subdivisé lui-même en deux patries, Hurons-Iroquois de l'Est et Tsimsyans-Haidas de la Côte NordOuest; un clan canadien, ensuite, qui pourrait tout aussi bien revêtir une double dimension, le folklore et les traditions, d'une part, et les tentatives de reconstitution historique, d'autre part. Le tout s'inscrivant sous le chapiteau totémique d'un intérêt remarquable pour l'art et la création. Il y a plus, cependant. Son œuvre contient, en effet, des récits qu'on hésite à recouvrir des catégories "roman" ou "fiction", mais qui les incarnent et les dépassent à la fois. The Downfall of Temlabam et Le Rêve de Kamalmouk ${ }^{1}$ appartiennent à cette geste dont on trouve très peu d'exemples dans la littérature canadienne. Il s'agit d'une espèce de pas de trois, tenant de la reconstitution, du récit et de la création, et que Barbeau utilisa pour tenter de subsumer à la fois la chronique anthropologique et le détour romanesque ${ }^{2}$.

Comme toutes les tragédies frontalières de l'âme et de la terre se déroulant aux limites incertaines des empires coloniaux en fermentation, l'action de ces récits est aussi simple à énoncer qu'impossible à bien circonscrire. En 1888, trois ans donc après l'assassinat politique du leader de la Résistance métisse, Louis Riel, se déroula un drame ressemblant fort à celui de la rivière Rouge, mais cette fois du côté de la Haute-Skeena, en actuelle Colombie-Britannique.

Attiré irrésistiblement par les Troncs-Pelés (ou les Dos-Blancs, comme on dit dans la Prairie) qui auront finalement raison de sa vie, Kamalmouk, chef Tsimsyan (Gitksan) du lac Guitoinkoul et des hauteurs de Temlaham - là où le mystère "d'une race intelligente et cultivée d'extraction mongole et tartare" côtoie ce pays envoûtant "de hautes montagnes, de forêts sombres et farouches, de lacs bleus comme le ciel, de rivières saumoneuses gonflées par un soleil d'été [venant miner] les pics neigeux et les glaciers éternels ${ }^{3}$ " - se voit bientôt entraîné dans des querelles et des meurtres qui feront éclater toute cohésion interne dans l'Arcadie autochtone. 
Par fidélité aux lignages et aux dieux ancestraux, Rayon-de-Soleil, fière épouse de Kamalmouk, se dissociera de son mari; le récit de Barbeau accompagne leur inéluctable divorce, ainsi que celui de la communauté entière aux prises avec une destinée dont elle ne "comprend ni la trame ni la trajectoire". Quand surviendront, quelques années plus tard, arpenteurs, géomètres et commissaires pour cadastrer le paradis perdu et encastrer la conscience sauvage, les magistrats auront beau jeu d'imposer leurs lois et leurs prévarications sur un terreau anthropologique perforé de toutes parts.

Si Kamalmouk et Temlaham désignent les deux versions, sinon les deux versants d'un même livre, il faut dire que les titres diffèrent passablement. "Temlaham" (pays de la bonne terre) renvoie,' en effet, au territoire et donc, à un être géographique matériel et spirituel; tandis que "Kamalmouk" est un homme, c'est-à-dire un territoire de chair, d'os et de culture. Tous ces personnages, et Barbeau insiste continuellement làdessus, sont entraînés et projetés dans des drames dont "ils ne saisissent ni le sens ni la portée", avant de devenir bientôt des "poussières perdues dans les annales de l'histoire". Mais qu'en est-il de l'auteur lui-même qui s'efforce passionnément de reconstituer de tels drames?

Je me plais à considérer Marius Barbeau comme l'un des ressortissants les plus doués de cette génération de Canadiens [Québécois, diraiton aujourd'hui, mais cela fait anachronique et irréell retours d'Europe ${ }^{4}$ qui imiteront, au départ, la méthodologie de leurs mậtres - nul n'est issu sans risque d'un monde colonial - avec un complet "trois-étages" en tweed écossais, pour ensuite trouver leur voie, souvent par des portages détournés. Comme ces rares sachems du Nord-Ouest qui auront su résister aux maladies, aux intempéries et... à la police montée, Barbeau aura connu une longue carrière qui le mènera à la fois au-delà de ses 85 ans (1883-1969) et de cette ethnologie stricto sensu dont il se réclamait pourtant entièrement.

La question qui se pose ici est la suivante: pourquoi Marius Barbeau n'a-t-il pas senti le besoin d'écrire le récit du Kamalmouk de sa Beauce natale ou celui du Sorcier de l'Isle d'Orléans? Parce que ce dernier n'existait pas ou parce que Barbeau n'a pas voulu le voir? En d'autres mots, la reconstitution lyrique du drame de Temlaham eût-elle été possible, pour Marius Barbeau, dans le cadre de son propre milieu canadien?

J'ai dû, pour ma part, parcourir mes premiers textes de Barbeau vers le milieu des années soixante, dans le Grand Nord, alors que les études et les travaux portant sur les mythes et autres histoires autochtones trâ̂naient dans les hangars et les officines des ministères des Ressources nationales, des Mines et Relevés techniques et où encore! Les Anglos appréciaient bien l'auteur des Mythes et des Pathfinders. Pratiquement tous les premiers écrits de Barbeau avaient été consignés dans leur 
langue; de plus, ayant étudié à Oxford, il leur appartenait donc un peu, en quelque sorte. Bref, il était un Native monté en grade qui s'était chauffé du même bois que ses maîtres, tout en conservant son odeur mentale caractéristique.

Ainsi Barbeau constituait-il, à leurs yeux, un French Canadian ayant réussi à sortir de la French Canadianness sans avoir subi trop de dommages in the making. Qui plus est, il faisait partie de la toute première fournée des anthropologues "autochtones" et, partant, il était donc susceptible de recéler quelque secret esssentiel. Secret qui jamais n'allait pouvoir être révélé directement aux plus hauts gradés de l'appareil académique, trop grande étant la distance ontologique les séparant de leur objet 5 .

Quelque temps plus tard, cependant, sous l'instigation d'un Jacques Rousseau ${ }^{6}$, j'avais parcouru un bref texte qui demeure toujours exemplaire: "La géographie de notre folklore ${ }^{7}$ ". Folk-Lore! Étymologiquement, le "lore " et le "folk", c'est-à-dire l'attraction et la séduction, d'une part, et la pensée domestique, d'autre part. Pensée domestique, c'est-à-dire la pensée apprivoisée et domptée plutôt que l'échappée légendaire et mythique.

Et pourtant, entre le folk et le mythe, il y a toujours une saga qui "percole". C'est peut-être là le secret d'un pays de Temlaham n'ayant pu être étendu jusqu'au pays de Canada (ou de Saint-Laurent). Pourquoi donc au juste? Je crois que la génération de Barbeau recherchait éperdument l'émergence d'une vérité qui ne fut pas la sienne. Et c'est pourquoi les "Kamalmouks" sont plutôt rares dans les annales géographiques du "chemin qui marche". Devant leur passé toujours présent, Barbeau et les premiers chercheurs du Canada (français) ne savaient peut-être pas qui ils étaient, mais ils ne voulaient surtout pas l'être.

À son retour des "vieux pays", le premier anthropologue du Canada aurait, paraît-il, déclaré ce qui suit: "Si tu veux réussir dans un tel domaine, oublie vite ta langue et mets-toi au travail ${ }^{8}$." Il n'allait pas respecter cette consigne pour lui-même, dois-je dire, mais lorsqu'on m'a rapporté ces propos pour la première fois, je les ai reçus avec emportement et forte agressivité. Pourquoi Barbeau, dont le talent et la persévérance touchaient au génie, eût-il dû se faire "anthropologue de service" comme ses ancêtres avaient été "truchements et interprètes" auprès de l'Amirauté terrestre? Aujourd'hui, cependant, je lui donne raison. Ses travaux sur la Côte Nord-Ouest, tout comme ceux portant sur les Hurons-Wyandots de la Vieille-Lorette ${ }^{9}$, n'auraient pu être publiés en français, à l'époque, j'en suis convaincu. Ni même conçus en français (canadien), quand on y réfléchit. Lorsqu'on appartient à un peuple minoritaire, les hauts faits des autres peuples minoritaires n'intéressent guère le secrétaire général de l'assemblée du rang d'en-bas.

En ces temps de political correctness, c'est à se demander par ailleurs si Barbeau eût été autorisé par la Présidence des Nations premières et le 
Secrétariat d'État à rédiger ce récit. Et, à la rigueur, si on lui eût concédé le droit de demeurer ce qu'il était: un homme en quête d'une histoire perdue et d'un mythe rédempteur, le Canada. Dans une étude intitulée "Marius Barbeau, ami de l'habitant et du sauvage ${ }^{10}$ ", Lotta Hitshmanova précise :

Marius Barbeau n'est pas seulement le grand spécialiste du Canada français. Il est presque incroyable qu'il ait pu, avec le même enthousiasme, le même dévouement scientifique, s'adonner à une tâche aussi difficile, aussi compliquée, peut-être plus responsable encore et plus exigeante : l'ethnologie des Indiens.

Pour ma part, je. suggérerais exactement l'inverse. Il était tout à fait normal qu'il le fit. Quant à savoir s'il était plus responsable et plus exigeant d'ausculter le Sauvage que le Canadien, c'est une question qui trouve sa résolution dans une juxtaposition qu'on a toujours refusé d'établir à la fois dans une telle ceuvre et un tel pays, à savoir que toute l'histoire identitaire de l'Amérique canadienne, c'est-à-dire pré-anglo, résulte de la rencontre sauvage/habitant.

Ainsi Marius Barbeau devint-il, presque trente ans.avant l'apparition des premiers anthropologues "natives-usa" assimilés-résistants et souvent d'origine franco ${ }^{11}$, le premier ethnologue d'un peuple jusque-là entièrement ethnologisé par les autres. Qu'allait-il faire? Il avait été formé pour anthropologiser les autres (ou, si l'on veut, ceux conçus comme autres) et non pas les membres de sa propre tribu. Il allait de fait l'avouer, en toute candeur, à une époque où le parcours anthropologique rejetait derechef tout métissage :

Le Dr Franz Boas, de New York, raconte-t-il, avait remarqué que les Indiens pratiquaient des contes français et espagnols. [...] À Lorette près de Québec, les Sioui m'avaient offert les récits de [...] P'tit-Jean tue le géant, et de L'Eau de la fontaine de Paris. Sur ce, le professeur Boas, en tant que sommité en la matière, me pria instamment de retourner à Lorette et de recueillir ces récits que j'avais refusés parce qu'ils n'étaient évidemment pas peaux-rouges ${ }^{12}$.

Voilà donc que Boas lui-même, son collègue d'études à Oxford, lequel deviendra, par la suite, l'un des pères fondateurs de l'ethnologieusa, allait pousser Barbeau à s'intéresser au folklore canadien, jusque-là l'apanage à peu près exclusif de chercheurs étrangers. Commencèrent alors une fouille et une collecte systématique des archives géographiques de ce peuple sans histoire et sans littérature appelé canadien. Il allait en sortir l'une des ceuvres maîtresses des Amériques, qui n'a pas été réexplorée depuis et qui contient l'un des fondements essentiels de l'identitaire canadien ${ }^{13}$.

Mieux vaut cependant laisser parler ici l'intéressé, tant il exprime avec justesse et acuité une assertion de la plus grande actualité :

La Nouvelle-France ayant recouvert dès les débuts de notre histoire la majeure partie de notre continent, le folklore de France fut le premier à se 
mélanger à celui des sauvages. Ceux-ci ne manquèrent pas tôt de s'y intéresser et d'en assimiler certains traits, d'autant plus que les missionnaires s'efforçaient de les civiliser et que les coureurs de bois ne manquaient pas de les débaucher de leurs grivoiseries. C'est pour cela qu'un certain nombre de contes français émaillent encore le répertoire indigène $[\ldots]^{14}$.

Difficile de savoir, avec le recul, qui, du porteur de croix ou du coureur en mocassins, aura été le plus grivois et le plus grand débaucheur de l'esprit! Mais ce que Marius Barbeau avance ici, il aura fallu attendre des décennies et toute l'autorité d'un Lévi-Strauss pour en prendre pleinement conscience ${ }^{15}$. Ainsi certains des traits culturels fondamentaux du Québécois contemporain font-ils pleinement partie du bagage ethnographique autochtone, et cela, aussi bien à l'est - au vieil-est maritime - qu'à l'ouest du continent - le grand-ouest et le grand large sans fin de cet océan terrestre appelé "Grand Nord". On comprend mieux alors, dans le contexte géopolitique et symbolique récent, combien certains des Autochtones les plus violemment anti-Québécois sont déjà des Québécois dans l'âme qui se refusent et vice versa ${ }^{16}$. C'est là une histoire de frères de sang ennemis dont Barbeau donnait déjà des clefs de lecture, il y a plus d'un demi-siècle. Pour cette seule raison, son ouvre mérite d'être entièrement réinvestie :

Pourquoi n'avait-on jamais prêté l'oreille, à Montréal et à Québec, à ces récits féeriques [et à ces contes franco-métis] qui, un peu partout, amusaient les gens à la campagne et même à la ville et qui étaient typiques des veillées en chantier [...], autant sur le parcours du Saint-Laurent qu'en régions maritimes? Oui, pourquoi? Impossible de répondre à cette question, si ce n'est que les Canadiens en ville ont toujours professé un certain mépris pour les "habitants" et les illettrés des campagnes et des bois. Et les contes rustiques étaient indignes de la littérature, qu'on importait d'ailleurs toute faite de Paris ${ }^{17}$.

Évidemment, c'est en métamorphisant de semblables contos rústicos que la littérature latino aura connu le boom que l'on sait à Paris et ailleurs, dans les années soixante-dix et quatre-vingt. Maintenant, cependant que la littérature autochtone et les écrits portant sur les sauvages du Nouveau Monde sont en train de connaître une popularité sans précédent, précisément en France ${ }^{18}$, qu'en est-il de l'œuvre d'un Barbeau ${ }^{19}$ ?

À vrai dire, lorsque j’ai pensé initialement à ce texte, j’étais convaincu que le rêve de Kamalmouk me conduirait directement au rêve jamais formulé de Marius Barbeau. Sans vouloir jouer au psycho-géographe éconduit, je n'en suis plus tout à fait sûr maintenant. Mais voyons d'abord ce que dit l'auteur de son roman, même s'il n'est pas pour autant le meilleur juge.

Les héros du récit, bons ou mauvais, inspirés ou fourvoyés, chasseurs ou sorciers, hommes ou femmes, et les deux principaux personnages, Rayon-de-Soleil et Kamalmouk, sont tous entraînés dans une chaîne de destruction fatale dont ils n'arrivent pas à se sortir. Et le rêve de Kamalmouk procède d'un tel enchevêtrement touchant au drame épique 
précolombien projeté dans la tragédie classique au milieu des forêts "sous attaque " de la Côte Nord-Ouest!

$\grave{A}$ travers une tentative de reconstitution lyrique qu'il n'allait guère répéter dans ses autres écrits, Barbeau s'en est expliqué avec grande clarté dans un avant-propos qu'il importe de citer in extenso:

Dès l'arrivée de l'Européen en Amérique [...], un conflit de races s'est engagé entre l'envahisseur et l'indigène. Ce conflit sans trêve ni merci devait entrấner la ruine du plus faible. Le Peau-Rouge [...] a fatalement glissé sur la pente qui conduit à l'abîme. Il n'existe plus, de notre temps [années trente et cinquante...], qu'à l'état déchu de métis ou de créole, emmuré en des "réserves", rongé par la maladie ou la mélancolie.

$[\ldots]$

La tragédie de Kamalmouk [...] présente un exemple typique de ce conflit, qui est presque de notre temps.

La vérité foncière du récit prend ici une allure lyrique; elle devient plus frappante que si elle eût côtoyé de trop près le terre à terre. Afin de l'enrichir, j'ai puisé largement dans [...] des traditions et des chants rituels que j'ai recueillis par centaines... Dans le développement de réminiscences fidèles, j'ai reconstitué par l'intuition et par le style le combat d'âmes étrangères, mais singulièrement humaines.

[...]

Oui, c'est la vérité même qui jaillit de ces pages empruntées aux annales indigènes, vérité qui s'amplifie dans une composition et une langue propres à nous. Ainsi redite, elle s'adresse à notre conscience hautaine [...] d'homme blanc qui se juge supérieur au reste de l'humanité.

Pourquoi faut-il que l'état de métis conduise inexorablement à la déchéance? Cela n'est jamais expliqué tant la chose semble implicite pour la pensée puriste. En fait, Barbeau apparaît ici, faut-il le souligner, comme un Blanc à plein temps, plutôt que Métis à temps partiel ou Créole canadien. Il demeure, à ses yeux, un scientifique qui raconte. Je crois, pour ma part, qu'il n'aurait tout simplement pu écrire ce livre s'il n'avait procédé et de l'un et de l'autre, c'est-à-dire du Métis et du Créole. En se présentant pourtant étranger au monde qu'il décrit, monde qu'il peut rejoindre, par ailleurs, par le talent et l'humain - l'humain qui repose en lui, Beauceron et anthropologue - , Barbeau joue un in-and-out qui l'honore. Moins out, cependant, qu'il ne le prétend, et plus in qu'on ne l'imagine.

Un style narratif d'une grande beauté, traversé d'envolées symboliques alternant entre le chant et la plainte, parcourt comme une brise le récit de Kamalmouk et nimbe les gens du pays de Temlaham d'une brume envoûtante. "Leurs chants, de dire Barbeau, descendent des temps anciens qui s'enveloppent dans une nuit poétique" (p. 36) ${ }^{20}$.

Ma trace, dans la neige fraîche, est blanche comme celle de la gélinote. Qui franchira après moi la brèche dans la voûte d'azur, à la poursuite du mirage infini? Je vais marcher dans le sentier du soleil, et ma voix est le grand vent qui secoue les branches sur les arbres du ciel. (p. 34) 
Qui suivra mes pas dans les sentiers de la vie, mes pas ensoleillés l'été, assombris l'hiver? Qui parcourra avec moi les vallons, portera le faix, et remontera la pente rude jusqu'à la faille dans le firmament? Qui pénétrera dans le mirage étoilé de l'au-delà? (p. 62)

-Triste oiseau de passage, tu arraches tes propres plumes", déclare Rayon-deSoleil. (p. 23)

Les noms des ancêtres sont bien plus nombreux que les têtes nouvelles qui pourraient les porter [et] les épidémies dévorent jeunes et vieux plus vite que les âmes n'ont le temps de renaître dans le ventre des femmes. (p. 25)

Ceux que je connaissais depuis longtemps disparaissent et ne sont pas remplacés. Mes voisins [...] ont-ils blanchi leur peau, pour mieux singer les Troncs-Pelés? Ont-ils oublié les esprits de la nature, ont-ils renié les ombres de leurs proches défunts? Ont-ils tous été baptisés dans l'eau, pour se repentir comme les chrétiens du péché de leur naissance?

Ayou-oa, ô ma vie! [...] Ma chanson est finie [...], mon temps est achevé, le temps d'un aîné qui a survécu à sa postérité. Mes membres défaillent sous le poids d'une souvenance sans lendemain [...]. Les vers s'acharneront à dévorer la dépouille que nous aurons refusée aux flammes de l'air, aux étoiles du firmament. (p. 108)

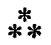

Le récit se termine sur une note d'un pessimisme absolu et d'un anéantissement total :

- Hay hâmidé! Ils m'ont laissé seul, tout seul. L'oubli les a éloignés de moi [...]. Ils ont méprisé ma détresse, pendant que je languissais, les mains vides, en proie à la faim, à l'angoisse [...]. En marchant, je me heurte aux arbres, que je prends pour des ombres. Toute ma vie a été une amère désillusion. Mon rêve fut une lame à deux tranchants. Ma fantaisie ne pouvait avoir de lendemain. La route tracée sur le sol ancestral n'était pourtant pas difficile à suivre [...]. J'ai eu le tort de m'en aller seul dans la jungle de l'imprudente nouveauté. J'ai tout connu, j'ai tout fait et, comme tant d'autres, j'ai moi-même tué l'homme.

Oh! pourquoi suis-je né, moi [Kamalmouk] qui n'aurais jamais dû voir le jour!

Autant que son héros, c'est Marius Barbeau qui parle à travers ces lignes empreintes d'une dignité désespérée. Mais d'où tire donc son origine une aussi profonde culpabilité chez Kamalmouk? Harcelé par sa femme Rayon-de-Lune, alias la Loutre-qui-Séduit, qui le poursuivait de ses sarcasmes devant son impuissance à venger ses fils emportés par la petite vérole, Kamalmouk a finalement assassiné Sorcier-Mauvais qui lui avait jeté un sort. Houspillé et ostracisé par tous, et surtout par lui-même, il se réfugie alors dans la forêt secrète jusqu'à ce que, hanté par le remords et l'ennui, il décide de se livrer au Blanc-Tronc-Pelé. Mais voilà que celui-ci l'assassine à son tour, en faisant porter le crime sur le sorcier lui-même dont les imprécations se seraient transformées en balle de fusil ["Un 
maléfice jeté par un sorcier tue aussi sûrement qu'une flèche tirée de l'arc" (p. 83)]. De toute façon, ces Saiouaches ${ }^{21}$ ne valent même pas l'air qu'ils respirent et encore moins la corde pour les pendre!

Du récit de Barbeau se dégage une détresse totale enveloppée dans un linceul géographique d'une beauté absolue. Dans ce monde de rivières riantes et de montagnes giboyeuses, chaque sentier est un récit et chaque récit... un hymne que viennent impitoyablement détruire les compagnies forestières et les agences de troc.

Le passé ne pouvant plus servir à tramer l'avenir et à rédimer un univers ne parlant plus la même géographie, Kamalmouk avait compris. "Je serai un Blanc, ou j'en mourrai" (p. 178), s'était-il promis. Eh bien!, les Blancs refuseront qu'il devienne un des leurs et il n'échappera pas à la mort pour autant, exécuté pour avoir osé vouloir leur ressembler tout en continuant d'adhérer aux lois de la tribu. "Rien ne pouvait les empêcher de donner une bonne leçon de justice aux sauvages arriérés qui [...] leur accordaient l'hospitalité." (p. 146) Ainsi Kamalmouk, lui qui était "si friand de la société des hommes et du secret des femmes" (p. 170), paiera-t-il de sa vie sa volonté d'adaptation aux Blancs et son inévitable trahison à l'endroit d'un monde déclaré obsolète, le sien:

Notre esprit reste confondu au milieu des écueils; notre sang même a perdu sa pureté. Les Troncs-Pelés en sont la cause, mais nous ne leur avons pas fermé la porte, quand nous le pouvions encore. Aujourd'hui, il est trop tard. [...] De même que les eaux de notre propre rivière Ségyukla se mêlent ici, sous nos yeux, à celles du Ksan, ainsi nos coutumes et notre sang doivent irrésistiblement se mêler à ceux des Blancs. (p. 113)

Qui soufflera le duvet de la réconciliation (p. 123) [...] sur la terre harcelée de.Temlahan? (p. 129)

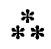

"Perdu à la fourche de sentiers vagues, concluera Barbeau, Kamalmouk ne savait encore où orienter ses pas de nomade infortuné: vers la civilisation, qui avait toujours été son rêve, ou vers le ya-ok, où il ne restait plus d'espoir?" (p. 132) Mais avant que Rayon-de-Soleil, Kamalmouk et les siens ne deviennent tout à fait les Fanny Johnson et les Jos Blow que leur conversion forcée aura amenés pour désigner leur identité dans les registres du christianisme et de la Compagnie de la Baie d'Hudson, hâtons-nous de rappeler ici leur nom d'origine.

$\mathrm{Au}$ moment où l'on s'efforce, en effet, de reconstituer, depuis Montréal, la bibliothèque du Nouveau Monde, version canadienne, qui eût cru qu'il eût fallu s'adresser un jour, pour en savoir un peu plus sur cette littérature orale et ce monde nouveau dont nous procédons, à tous 
ces personnages qui apparaissent chez Barbeau. Héros évaporés et écrivains de l'air dont les noms de plume - plume-d'aigle et plume-de-vie, s'entend - s'énoncent comme suit:

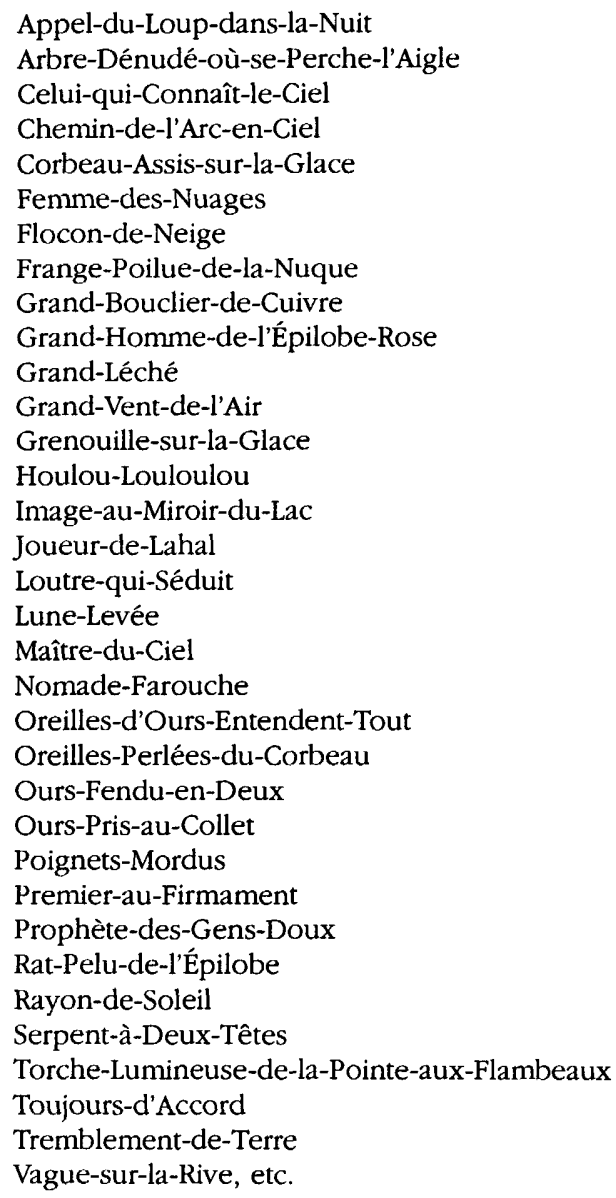

Dans un hommage lumineux récemment rendu à Marius Barbeau sous le titre "Ce regard qui nous rendit la mémoire", Fernande SaintMartin ${ }^{22}$ reprend une formule que l'auteur du Rêve de Kalmamouk se plaisait, insiste-t-elle, à répéter: "Nous sommes des ombres et nous nous en irons comme des ombres." En fait, cette idée est empruntée directement à Kalmamouk lui-même qui répétait, titubant: "En marchant, je me heurte aux arbres que je prends pour des ombres. Hadzâh!" 
Mais pourquoi donc Kalmamouk, alias Oreilles-Perlées-du-Corbeau chez les siens, alias Jim - Guitoinkoul Jim - ou "Viens ici, Jim, maudit Sauvage" chez ses bons amis, les Troncs-Pelés, devait-il être implacablement condamné? "On accepte tout d'un vainqueur, sauf la honte", dira Barbeau (p. 192). Ainsi, dans la lucidité de son dernier chant, la mort apparaît-elle, pour Kamalmouk, comme la seule alternative possible à la honte et la déchéance.

Je m'effarouche... mon gosier se serre, au souvenir des visages humains que j'ai connus $[\ldots]$ troublants dans leur duplicité, inconscients même de leur destinée.

Je m'effarouche lorsque ma pensée prophétique traverse l'arc-en-ciel, le pont des âmes en route pour l'au-delà [...] qui retentit de lamentations.

Je m'effarouche lorsque les jours monotones frôlent les uns après les autres mon front levé dans l'attente, mon front trop souvent voilé de brouillards.

N'as-tu pas honte de t'abandonner si lâchement à ton angoisse [...] sur le lit de mousse des nuits d'été? Pourquoi rechercher la solitude, lorque la double voix des accouplements vibre à ton oreille et grise les sens d'une longue ivresse. [...] Ne sois plus triste au milieu de tant d'exubérance!

Soleil, fleurs, fruits, hymen et chants clairs, n'en voilà-t-il pas assez pour te faire sourire, homme aux inépuisables chagrins, né en pleurant de la femme et incinéré après la mort au milieu de funèbres complaintes? Si cette vallée pour toi doit être un cimetière, que tes cendres se changent en poudre d'or sous un soleil éternel! (p. 171-174)

Le paradis perdu des indigènes de la Skeena s'est enveloppé d'une brume aussi légendaire que celle de la genèse babylonienne, conclut Barbeau. Son nom ne figure même pas à la page des récriminations multiples des races vaincues qui ont sombré dans les remous du passé. Kamalmouk appartient désormais aux ombres.

Je n'en suis pas sûr du tout.

Grâce à Barbeau-Marius, justement, Kalmamouk sera à jamais sorti des ombres emportées par les archives du vent.

Mais, au fait, à l'époque où, sous l'influence de la géographie vernaculaire, les Canadiens se donnaient tous des surnoms - "Marsouind'Eau-Douce ", "Grande-Langue-du-Ruisseau-Jureux", etc. - - je me demande comment se serait appelé Barbeau? "Poisson-d'Eau-Douceà-Chair-Estimée", suggère le dictionnaire, sous le substantif "barbeau " pour ne s'en tenir qu'à un terre à terre nominatif sans aucun respect pour l'invocation autochtone. Mais non, un tel nom ne va pas du tout; nous savons pourtant tous qu'il n'y a pas d'ombre sans lumière!

Alors, voilà. Que soit salué à jamais comme l'un des grands écrivains de ce continent, celui qui, par fidélité à son œuvre, il faudra bien appeler un jour: "Lumière-que-Porte-l'Ombre".

Ou, mieux encore : "Rêve-qui-Surgit-de-l'Ombre". 
1. Rédigé directement en anglais, dans un langage aussi riche que sonore (Marius Barbeau était, comment dire, "parfait bi-scripte "), et publié en 1928 [Toronto, Macmillan], The Downfall of Temlaham se verra décerner le prix David, section anglophone. Ce n'est que vingt ans plus tard [Montréal, Fides, 1948] que Barbeau publiera, sous un titre assez différent - Le Rêve de Kamalmouk - une version légèrement remaniée du titre précédent pour laquelle il n'obtint aucun prix. Du moins, pas encore. Question de prix littéraires, je ne peux résister à la tentation de faire ici un parallèle avec l'ouvrage, également "bi-scripte", de Nancy Huston, Le Cantique des Plaines [Paris/Montréal, Actes Sud/Leméac, 1994], primé à Ottawa (prix du Gouverneur général, section francophone) et qui a suscité une telle polémique. La version anglaise, The Plain Song lque je re-traduirais par "La chanson plate", le gros de l'action, après tout, ne se passe pas très loin au nord de la rivière Platte, au Nébraskal ne s'est vu octroyer aucun prix. Du moins, pas encore, là non pius.

2. Le Rêve de Kamalmouk constitue un récit autonome dans lequel on ne retrouve pas les sources d'information accompagnant la version originale anglaise. The Downfall of Temlaham a été réédité chez Hurtig à Edmonton, en 1973, avec une introduction de George Clutesi, et suivi de trois narrations [The Retaliation of Keemelay, The Painted Goat of Stekyawden et The Downfall que Marius Barbeau n'avait pas cru bon traduire, semble-t-il, pour les inclure dans la version française de sa narration.

3. Extraits de l'avant-propos.

4. La double Europe de Londres et de Paris, bien sûr. La belle-mère-patrie, d'abord; la mère-patrie, ensuite.

5. Je ne peux m'empêcher d'ouvrir ici une parenthèse. On a oublié jusqu'à quel point le F.C. (pour employer le sigle en vigueur) éclairé - c'est-à-dire le French Canadian légèrement et suffisamment assimilé, pour ne pas dire culturellement faisandé, mais conservant toujours un peu de sa pensée vernaculaire - était considéré dans tout l'Empire comme une pièce rare dont il fallait cultiver à tout prix le commerce, si elle était fidèle. Les Anglos du Commonwealth, gestionnaires des autochtones et autres sujets, ont toujours procédé par truchement et interprète. De tous les fils minorisés de l'Empire, le F.C. leur apparaissait donc un interprète d'autant plus prometteur qu'il était issu de leur toute première colonie exogène, le Canada, et qu'il pouvait généralement passer pour Blanc, malgré ses origines ambiguës. De là l'extrême importance des premiers diplômés "frenchés en sciences sociales. Natifs eux-mêmes, ils pouvaient servir d'interprètes lettrés à la pensée native des autres. Jacques Ferron a tellement bien compris cela, lui qui, dans un de ses contes, renvoie à l'Empire la monnaie de sa pièce, en dépêchant l'un de ses personnages au British Museum ou je ne sais où, afin de poursuivre ses travaux de terrain et ses auscultations crâniennes chez les Natives britanniques impériaux eux-mêmes.

6. Jacques Rousseau, cet autre demi-inconnu de l'écriture-canada, qui parlait volontiers de Barbeau. Peut-être avaient-ils conjointement fait partie du Groupe des Dix, je ne connais guère ces détails.

7. Paru dans Mélanges géographiques canadiens offerts à Raoul Blanchard, Québec, Les Presses de l'Université Laval, 1959, p. 115-122.

8. C'est Robert Laplante qui m'a rapporté un jour une telle assertion dont j'ai oublié l'exacte formulation textuelle.

9. Incidemment, son étude de 1915, Huron and Wyandot Mythology, vient tout juste d'être traduite. Voir, sous la direction de Pierre Beaucage, Mytbologie huronne et wyandotte, Les Presses de l'Université de Montréal, 1994, XLV-X-439 p.

10. Culture, vol. I, $\mathrm{n}^{\circ} 3$, mars 1946, p. 66-71.

11. Je pense en particulier à des ouvrages tels Custer Died for Your Sins de Vine Deloria Jr., publié en 1965 [Il existe une traduction française de ce texte sous le titre un peu simpliste de Peau-Rouge, Paris, Édition Spéciale, 1972, 281 p.]. Incidemment, le nom Deloria est la transformation en hispano du nom franco-sioux canadien "Delors". Voir William Powers, "Les premiers contacts entre les Français et les Sioux: témoignage d'un ethnologue adopté par des Indiens ", Joëlle Rostkowski et Sylvie Devers, Destins croisés, Paris, Albin Michel/Unesco, 1992, p. 249-261. "Assimilé-résistant "! Qu'est-ce à dire? C'est 
là la condition ontologique de tout être métis qui ne réussit à exprimer sa résistance et sa culture évanouie-évanescente que dans l'assimilation aux forces phonétiques et politiques qui lui ont écorché l'âme. L'alphabétisation et la mise en écriture comme processus d'acculturation et de trahison sans lequel le secret géographique de la tribu serait à jamais emporté par la succession des saisons et le weathering académique!

12. Rapporté dans Mélanges géographiques canadiens offerts à Raoul Blanchard, op. cit., p. 115.

13. En complétant le travail de Barbeau par celui des récits et témoignages du missionnaire français, génial et conspué, Émile Petitot, chez les Métis dènès et ailleurs, se dégage peu à peu cette autre facette du sous-bassement franco en Nord-Amérique. Mais je doute quelquefois, pour ma part, que ce travail ne soit jamais rendu à terme, car il jette un doute aussi profond que bouleversant sur l'image identitaire que s'offre le Québec sur lui-même depuis toujours.

14. Mélanges géographiques candiens offerts à Raoul Blanchard, op. cit., p. 115.

15. Voir là-dessus "Le chat sauvage, le coyote et le québécois", Spirale, été 1992, p. 12 et aussi * Paroles de Québécois traduites du Tchippewayan et autres dialectiques géographiques ", Recberches amérindiennes au Québec, vol. XXII, nos 2-3, 1992, p. 117-122.

16. Évidemment, la catégorie d'assignation "Québec "fait ici problème, car elle fait appel à une frontière identitaire juridique interdisant toute expansion mentale et territoriale. Les Autochtones de la Prairie, dans leur immense majorité, aussi bien aux États-Unis d'Amérique qu'en Amérique britannique, le so-called Canada, ont du sang québécois qui circule dans leurs veines et leur imaginaire. Mais cela n'est pas avoué de part et d'autre, et je dirais même, caché avec violence, l'idée même de Québec empêchant de le faire.

17. Mélanges géographiques canadiens offerts à Raoul Blanchard, op. cit., p. 116.

18. Pourvu qu'elle soit traduite de l'américain [sic], doit-on s'empresser d'ajouter.

19. Laquelle connaît, incidemment, un grand regain de popularité chez les chercheurs d'Américana et de Canadiana de l'Anglo-Amérique, mais demeure entièrement inconnue dans le monde francophone.

20. Dans cette citation et celles qui suivent, les chiffres entre parenthèses renvoient à la page dont elles sont extraites, clans l'édition publiée chez Fides.

21. Déformation du mot canadien * sauvage * ou *chavage " qui aura passé ainsi clans le jargon "chinouk" de la Côte Nord-Ouest, lequel constitue, avec le Métchiff, la contribution essentielle et exclusive de la culture québécoise à la littérature orale des Amériques.

22. Publié dans Les Écrits, no 83, 1995, p. 21-29. 